\title{
Development of Functional Topography in the Corticorubral Projection: An In Vivo Assessment Using Synaptic Potentials Recorded from Fetal and Newborn Cats
}

\author{
Wen-Jie Song and Fujio Murakami \\ Division of Biophysical Engineering, Graduate School of Engineering Science, Osaka University, Toyonaka 560, Japan
}

In mammals, topographic maps emerge from initially diffuse projections during development. To gain insight into the mechanisms governing the transition from a diffuse projection to a topographic map, we studied topographic specificity of functional connections during development, using the cat corticorubral system as a model. In the adult cat, rubrospinal neurons in the dorsomedial part of the red nucleus $(R N)$ receive input primarily from the forelimb area of the sensorimotor cortex, whereas those in the ventrolateral part receive input primarily from the hindlimb area. During development, axons from the sensorimotor cortex arrive in the RN at embryonic day 50 (E50) (Song et al., 1995a) and are diffusely distributed in the RN until postnatal day 13 (P13) (Higashi et al., 1990). Here, we studied the development of the pattern of functional cortical inputs to individual rubrospinal neurons, using synaptic potentials recorded in vivo. The functional topography in each rubrospinal neuron in developing cats was examined and classified

Topographic organization in neuronal connections provides a structural basis for parallel processing in the brain. The development of topographic maps, therefore, has been a subject of intense research (for review, see Udin and Fawcett, 1988; Gierer and Muller, 1995; Roskies et al., 1995). In order for a topographic map to form, growth cones must navigate along precise pathways, find their target, and then make synapses with appropriate neurons in the target. The central issue in the formation of topographic maps is, therefore, how axons find input-recipient cells in their target region.

This issue has been extensively studied in the retinotectal projections of fish and amphibians. In these species, retinal axons are guided or restricted to the topographically correct tectal regions without errors from the outset of innervation (Sakaguchi and Murphey, 1985; Stuermer, 1988). Interactions between retinal axons and tectal neurons via position-specific molecules have been suggested to be involved in such precise guidance (for review, see Stirling, 1991; Roskies et al., 1995; Drescher et al., 1997).

Received Jan. 27, 1998; revised July 20, 1998; accepted Aug. 28, 1998.

This work was supported by Grants-in-Aid for Scientific Research on Priority Areas (7279101 and 9280217) and Grants for Young Investigators (9780769) from the Ministry of Education, Science, and Culture of Japan. The authors are investigators of Core Research for Evolutional Science and Technology. We thank Drs. N. Yamamoto and E. S. Ruthazer for discussion and suggestions on this manuscript, M. Kanda for the kittens, and K. Okawa and T. Ohno for help in some of the experiments.

Correspondence should be addressed to Wen-Jie Song, Division of Biophysical Engineering, Graduate School of Engineering Science, Osaka University, Machikaneyama 1-3, Toyonaka 560, Japan.

Copyright (๑) 1998 Society for Neuroscience $0270-6474 / 98 / 189354-11 \$ 05.00 / 0$ either as adult-like or nonadult-like by comparison with the adult pattern. In preterm kittens from E61 to E65, only about half of the recorded neurons $(41 \% ; n=22)$ showed adult-like functional topography. This percentage, however, increased to $82 \%(n=56)$ in P1-P8 kittens and to 93\% $(n=42)$ in P13-P28 kittens. These results, in conjunction with the above mentioned anatomical observations, suggest that corticorubral axons make functional synapses nonselectively with rubrospinal neurons before birth. Furthermore, the functional topographic map developed earlier than the anatomical map ( $<$ P8 vs $>$ P13), suggesting that there is a developmental step of selective promotion of synapse formation and/or selective enhancement of synaptic efficacy in topographically appropriate regions in the RN, before the emergence of the mature anatomical map.

Key words: topographic map; sensorimotor cortex; red nucleus; rubrospinal neuron; immature synapse; intracellular recording

Studies of the rodent retinocollicular projection, however, suggest that a different cellular mechanism operates for the formation of topography; retinal neurons initially send diffuse projections to the superior colliculus, and the topographic map is formed only thereafter (Simon and O'Leary, 1992a,b). In vitro, however, retinal axons at stages corresponding to the diffuse retinocollicular projections respond to position-specific cues in the colliculus, preferentially forming branches on membranes derived from topographically appropriate regions (Simon and O'Leary, 1992b; Roskies and O'Leary, 1994). Moreover, the nature of the tectal cues resembles that in the lower vertebrate; temporal retinal axons are repelled by the caudal tectum, whereas nasal axons can grow on both the rostral and the caudal tectum (Walter et al., 1987; Godement and Bonhoeffer, 1989; Vielmetter and Stuermer, 1989; Simon and O'Leary, 1992b). These studies may suggest that rodent retinal axons in vivo initially ignore position-specific cues in the target. Alternatively, rodent retinal axons may also respond to the cues in vivo but by selectively forming synapses with cells in topographically appropriate regions at an early stage of development, when anatomical arrangement of topography is not evident. A test of such ideas requires analyses of the distribution of synapses, but so far most studies on the development of topographic maps have focused on the development of axonal morphology and little attention has been paid to the development of synaptic connections.

We have examined the perinatal development of topographic specificity of "functional" connections, or functional topography, in the corticorubral system of the cat, using intracellularly recorded synaptic potentials. The corticorubral system of the cat is 
also organized in a topographic manner; rubrospinal neurons in the dorsomedial part of the red nucleus (RN) receive inputs primarily from the forelimb area of the sensorimotor cortex, innervating the cervicothoracic cord, whereas those in the ventrolateral part receive inputs primarily from the hindlimb area, projecting to the lumbosacral cord (see Fig. 1A) (Pompeiano and Brodal, 1957; Mabuchi and Kusama, 1966; Tsukahara and Kosaka, 1968; Jeneskog and Padel, 1983). Like the retinotectal projection of the rodent, the corticorubral projection starts from a diff use pattern of innervation, followed by axon arborization in topographically appropriate regions, leading to the formation of a topographic map (Higashi et al., 1990). This system is ideal for studying the development of functional topography, because (1) a technique for intracellular recording in vivo from developing rubrospinal neurons has been established in our laboratory (Song et al., 1995b), and (2) the morphological development of corticorubral axons has been well documented; cortical efferents enter the RN at approximately embryonic day 50 (E50) (Song et al., 1995a) but do not show localized distribution in the RN until postnatal day 13 (P13) (Higashi et al., 1990).

The results presented here show that the adult-like functional topographic map in the corticorubral projection is not formed before birth, suggesting that cortical axons initially make functional synapses nonselectively with rubrospinal neurons. The functional topographic map, however, was found to be formed in postnatal cats before the emergence of a clear anatomical map.

\section{MATERIALS AND METHODS}

Animals. Five preterm and 36 postnatal cats aged E61 to P28 were used. All animals were raised in a breeding colony of Aburahi Labs of Shionogi \& Co., Ltd. Gestation period was $67 \mathrm{~d}$ on average. Mating was allowed for $12 \mathrm{hr}$, and the time of mating was counted as E0. For postnatal cats, the day of birth was counted as P0.

Surgery. All experiments were conducted in compliance with the Guidelines for Use of Laboratory Animals of Osaka University. Cats were anesthetized with initial doses of sodium pentobarbitone (Nembutal) of $25-30 \mathrm{mg} / \mathrm{kg}$ (i.p.). Supplementary doses of Nembutal (2-4 $\mathrm{mg} / \mathrm{kg} / \mathrm{hr}$, i.v.) were given regularly during surgery and recording to maintain anesthesia. Gallamine triethiodide $(20 \mathrm{mg} / \mathrm{kg} / \mathrm{hr}$, i.v. $)$ was applied to paralyze the animal during recording. The adequacy of anesthesia was judged by the absence of reflexes to ear and toe pinches before paralyzation. The procedures for delivery of the fetuses, fixation of the animal to the stereotaxic frame, and other procedures have been described previously in detail (Song et al., 1995b).

Stimulation and intracellular recording. The experimental arrangement is diagrammed in Figure 1, $B$ and $C$. The RN was located by recording orthodromic field potentials evoked by activation of the contralateral cerebellar nuclei (CN) (Song et al., 1993). A pair of acupuncture needles insulated except at the tips, was used as a bipolar electrode for the activation of the $\mathrm{CN}$. The needles had a diameter of $0.2 \mathrm{~mm}$, and the tips were exposed at a length of $0.3 \mathrm{~mm}$ under a dissecting microscope. The distance between the two tips of a bipolar electrode pair was $2 \mathrm{~mm}$. After microelectrode impalement, antidromic spikes in rubrospinal cells were evoked by stimulation of the first cervical cord segment (C1) and the first lumbar cord segment (L1) using bipolar silver ball electrodes. Cells responding only to the stimulation of $\mathrm{C} 1$ were referred to as $\mathrm{C}$-cells, and those responding to stimulation of both $\mathrm{C} 1$ and $\mathrm{L} 1$ were called L-cells (Tsukahara and Kosaka, 1968). The accuracy of this method for identification of RN neurons has been verified by intracellular staining (Song et al., 1993, 1995b; see below).

To assess the functional topography of the corticorubral projection, the sensorimotor cortex ipsilateral to the recorded RN was stimulated at four sites at the same strength (see Fig. 1C). The same type of bipolar electrodes as for the $\mathrm{CN}$ were used for stimulation of the sensorimotor cortex. The bipolar electrodes were lined perpendicularly to the cruciate sulcus and were set to straddle the sulcus to minimize mediolateral spread of stimulation currents. To compensate for developmental change of brain size, the length (L) of the cruciate sulcus was measured first. This length ranged from $1.7 \mathrm{~mm}$ to $5.8 \mathrm{~mm}$, depending on age. The third pair of electrodes from the midline was always set at the lateral end of the sulcus; the distance between pairs of electrodes was set at $(2.3 / 5) \mathrm{L}$. By this method, the medial two pairs of electrodes were placed in the hindlimb region, and the lateral two were placed in the forelimb region (Rubel, 1971; Jeneskog and Padel, 1983). For the preterm kittens, two pairs of electrodes were used because of the small size of the cortex. One pair was set at the midpoint between the locations where medial two pairs of electrodes would have been placed in the four-electrode arrangement; the other was set at the midpoint between the lateral two pairs. In a control experiment, three of the postnatal kittens were studied using two pairs of electrodes. Electrode tips were inserted into the cortex at a depth of $0.5 \mathrm{~mm}$ for kittens younger than 1 week and $1 \mathrm{~mm}$ for older kittens. A single voltage pulse with a duration of $80 \mu \mathrm{sec}$ was used for stimulation. Stimulus strength was read in voltage, but current flowing through the stimulation electrodes was checked before recording by measuring the voltage across a $10 \mathrm{~K} \Omega$ resistor connected to the stimulation circuit in series. The current did not vary substantially among electrodes $(<2 \%)$. Because cortical efferent neurons are distributed in a sheet, relatively strong stimulus strength was required to evoke a corticorubral response. The topography was estimated using stimulus strength of 30-100 V, corresponding to currents of $130-1000 \mu \mathrm{A}$. A wider range of $10-120 \mathrm{~V}$ was used to examine synaptic potentials.

The method of intracellular recording has been described previously (Song et al., 1995b). The voltage from the recording electrode was amplified by a preamplifier, displayed on an oscilloscope, and stored in a pulse-code-modulation video recorder. Signals in the recorder were digitized and analyzed off-line using a personal computer. In some early experiments, synaptic potentials were photographed from the oscilloscope and analyzed.

Intracellular injection of biocytin. Biocytin was injected intracellularly after recording in 14 cells to verify that recorded cells were in fact RN neurons. The methods for injection and visualization of biocytin followed Song et al. (1993).

Wheat germ agglutinin-horseradish peroxidase injection. To study the topography of the rubrospinal projection in fetal cats, wheat germ agglutinin-horseradish peroxidase (WGA-HRP) (2\% in saline, $0.1 \mu \mathrm{l}$; Toyobo) was injected into the L1 segment with a Hamilton syringe, under the anesthesia specified above. After $2 \mathrm{~d}$, the fetus was perfused under deep anesthesia with $1.25 \%$ glutaraldehyde and $1 \%$ paraformaldehyde in phosphate buffer. The brainstem was sectioned into $70-\mu \mathrm{m}-$ thick sections and processed for visualization of WGA-HRP using tetramethyl benzidine as the chromogen. Labeled cell bodies were marked on a drawing paper with a drawing tube attached to a light microscope.

Ibotenic acid injection. To verify that corticorubral cells are responsible for synaptic potentials evoked in rubrospinal neurons by stimulation of the sensorimotor cortex, cortical neurons were selectively destroyed by ibotenic acid injection (Schwarcz et al., 1979). In two P4 kittens, 2-2.5 $\mu \mathrm{l}$ ibotenic acid $(5 \mathrm{mg} / \mathrm{ml}$ in saline; Sigma, St. Louis, MO) was injected into each of four or six sites of the pericruciate cortex. The kittens were used for electrophysiological analyses $4 \mathrm{~d}$ after the injection. The extent of lesion in the sensorimotor cortex was examined in Nissl-stained sections after recording.

Histology. After electrophysiological experiments, the loci of stimulating electrodes in the $\mathrm{CN}$ were marked by passing negative current $(0.4$ $\mathrm{mA}, 5 \mathrm{sec}$ ), and the animal was perfused with $3.5 \%$ formaldehyde under deep anesthesia. Recording electrode tracks and stimulating electrode loci were verified in Nissl-stained sections.

Statistical analyses. The functional topography in the corticorubral projection was assessed in individual rubrospinal neurons and was classified either as adult-like or nonadult-like. The occurrence of cells with adult-like topography should then follow the binomial rule. With a sufficiently large number of cells, the binomial distribution approximates a normal distribution (Soeda et al., 1980; Lapin, 1983) of the form $z=$ $(r-n p) /(n p(1-p))^{0.5}$ where $z=$ normal variable, $n=$ total number of cells, $r=$ number of cells showing adult-like topography, and $p=$ theoretically predicted probability of cells showing adult-like topography. This equation was used to test whether the corticorubral connection was random (i.e., if $p=0.5$ ). To test whether the proportion of cells showing adult-like topography was significantly different between groups, the normal variable $z=\left(p_{1}-p_{2}\right) /\left(p_{0}\left(1-p_{0}\right)\left(1 / n_{1}+1 / n_{2}\right)\right)^{0.5}$ was used (Soeda et al., 1980), where $p_{1}$ and $p_{2}$ are the proportions of cells showing adult-like topography in group $1\left(p_{1}\right)$ and group $2\left(p_{2}\right)$ of animals, $n_{1}$ and $n_{2}$ are total numbers of cells in group $1\left(n_{1}\right)$ and group $2\left(n_{2}\right), p_{0}=$ $\left(n_{1} p_{1}+n_{2} p_{2}\right) /\left(n_{1}+n_{2}\right)$. 
Figure 1. Topographic map of the corticorubrospinal system of adult cat and the experimental arrangement for studying the development of the topography. A, A schematic depiction of the topography in adult cat. The forelimb area of the sensorimotor cortex, i.e., the lateral posterior sigmoid gyrus ( $L P S G)$, projects to the dorsomedial portion of the RN, which innervates the cervicothoracic cord. The hindlimb cortical area, the medial posterior sigmoid gyrus (MPSG), projects to the ventrolateral portion of the RN, which innervates the lower cord. Rubrospinal cells in dorsomedial and ventrolateral portions of the $\mathrm{RN}$ are called $\mathrm{C}$-cells and L-cells, respectively. $B$, The experimental arrangement. The RN was identified by stereotaxic coordinates and field potentials evoked by stimulation of the contralateral deep CN. Rubrospinal neurons were identified either as a C-cell or an L-cell by their responses to stimulation of the first cervical segment (C1) and the first lumbar segment (L1). Synaptic potentials evoked by stimulation of different sites in the cortex were recorded intracellularly from rubrospinal neurons to assess the pattern of functional input from the cortex to the recorded neuron. $C$, A dorsal view of the cortex showing the locations of the stimulating electrodes in the sensorimotor cortex. Bipolar stimulating electrodes were set at four sites for postnatal kittens and two for fetal cats. The medial two pairs of electrodes were set in the hindlimb region, and the lateral two pairs were set in the forelimb region. See Materials and Methods for details. All pairs of the bipolar stimulating electrode were lined perpendicularly to the cruciate sulcus $(C S)$ and were set to straddle the sulcus to minimize current spread to neighboring sites.
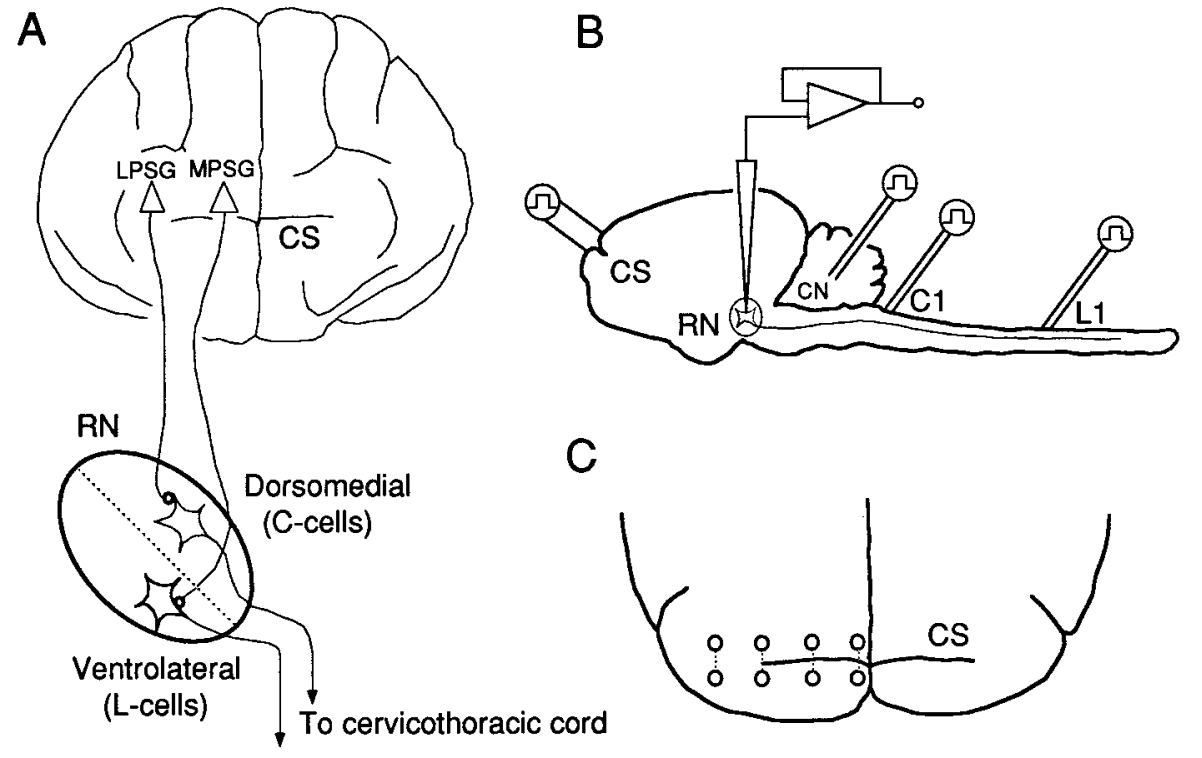

ro cervicothoracic cord

To lumbosacral cord

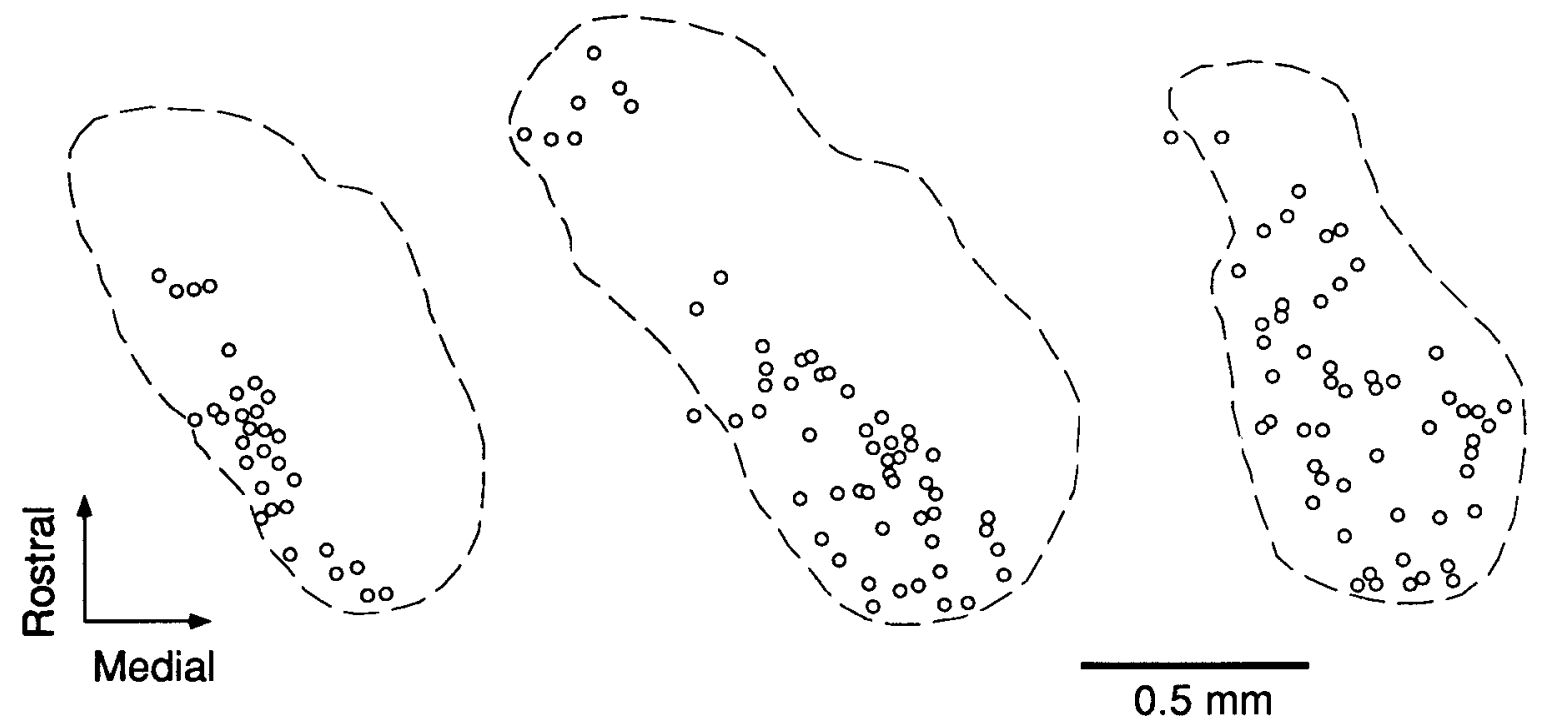

Figure 2. The topography of rubrospinal projection in fetal cats. The distribution of rubrospinal neurons retrogradely labeled by injection of WGA-HRP into the first lumbar segment in an E61 kitten. Each circle represents a labeled cell body, and the broken line approximates the border of the RN. The third, the fifth, and the seventh sections of eight horizontal sections, counting from dorsal end of the RN, are lined from left to right. Note the strict localization of labeled neurons to the ventrolateral portion of the RN.

\section{RESULTS}

\section{Topography in rubrospinal projections of preterm kittens}

Because rubrospinal neurons in the dorsomedial part of the RN project to the cervicothoracic cord and those in the ventrolateral part innervate the lumbosacral cord (Pompeiano and Brodal, 1957), the location of a rubrospinal cell within the RN can be inferred from its response to stimulation of the spinal cord; cells responding only to stimulation of the $\mathrm{C} 1$ spinal segment should be in the dorsomedial portion (C-cells) (see Materials and Methods), whereas cells responding to stimulation of both $\mathrm{C} 1$ and L1 segments should be in the ventrolateral portion (L-cells) (Fig. $1 A)$. Although the topography in the rubrospinal system is observed in young kittens (Pompeiano and Brodal, 1957), it is not known when this topography is established. To study the functional topography in the corticorubral system of perinatal cats, it is necessary to know whether the location of a rubrospinal neuron within the RN in developing cats can also be inferred by their antidromic responses. We thus set out to study the topography in rubrospinal projections in prenatal cats. Figure 2 illustrates the distribution of retrogradely labeled neurons within the RN at E61. The neurons were retrogradely labeled by WGA-HRP in- 

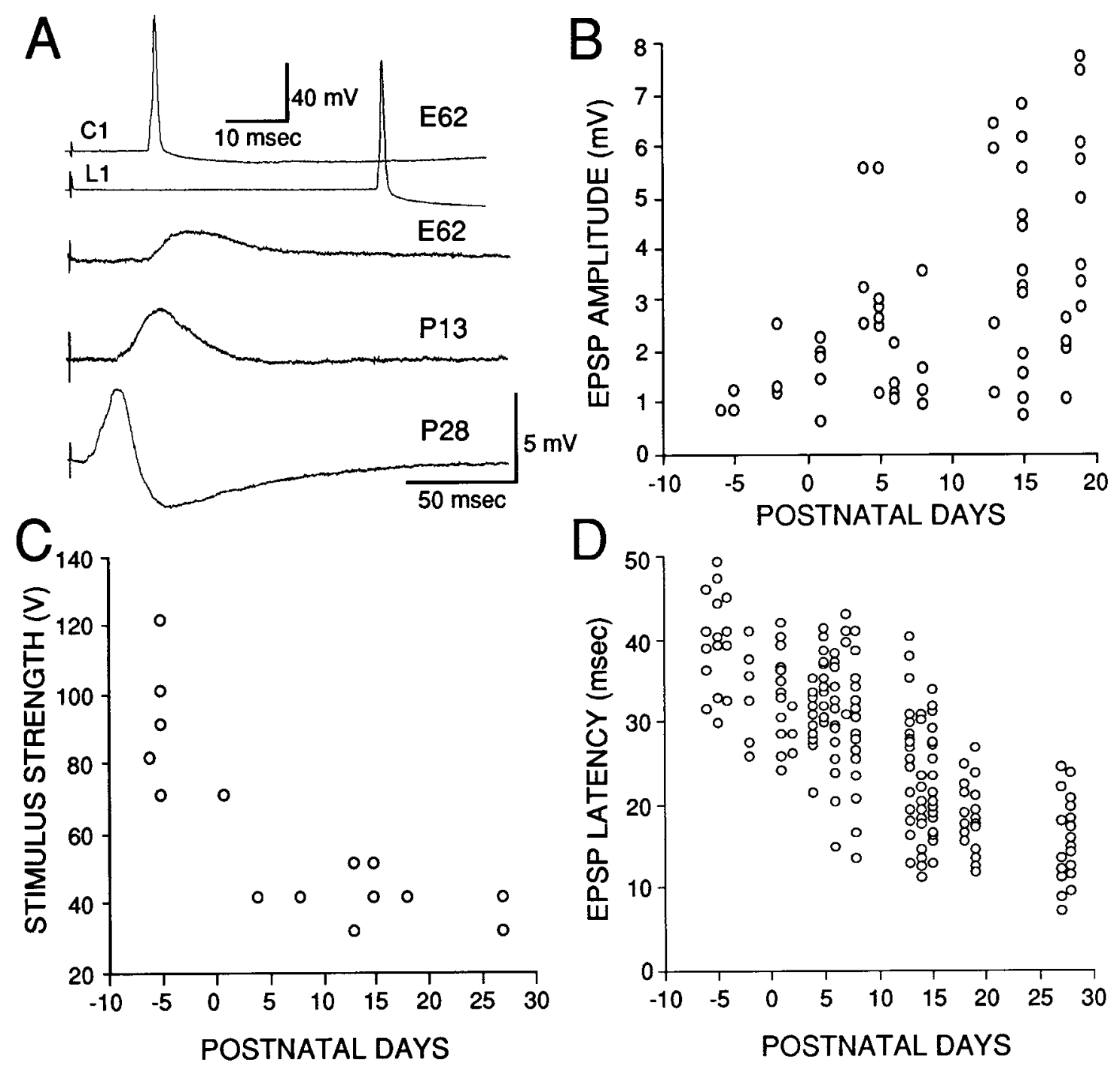

Figure 3. Synaptic potentials evoked in rubrospinal neurons by stimulation of the sensorimotor cortex in perinatal kittens. A, Top two traces, Antidromic responses evoked in an E62 neuron. Such antidromic spikes were used for identification of rubrospinal neurons as C-cells or L-cells. Traces three to five are examples of synaptic potentials evoked in an E62 fetus, a P13 kitten, and a P28 kitten, respectively. The corresponding stimulus strengths are 80 V, $40 \mathrm{~V}$, and $40 \mathrm{~V}$, respectively. $B$, Plot of the amplitude of EPSPs evoked by a $50 \mathrm{~V}$ stimulating pulse as a function of postnatal days $(n=56)$. $C$, Plot of the stimulus strength required for evoking an EPSP with an amplitude of $1.2 \pm 0.2 \mathrm{mV}$ as a function of postnatal days $(n=15)$. $D$, Plot of the latency of the EPSPs as a function of age. The latency of all EPSPs evoked from topographically appropriate cortical sites in 128 recorded neurons was plotted. The latency of EPSPs having slow rise from the baseline was difficult to determine and was excluded.

jected into the L1 segment of the spinal cord. Figure 2 clearly shows that neurons projecting to or beyond the L1 segment were located in the ventrolateral portion of the RN. This topographic arrangement was absolute; not a single neuron was labeled in the dorsomedial portion. Similar results were obtained in another E61 fetus and an E63 fetus.

Intracellular staining of electrophysiologically identified rubrospinal neurons revealed that all C-cells $(n=6$; one from an E62 fetus) were in the dorsomedial portion of the RN, and all L-cells $(n=8)$ were in the ventrolateral portion (data not shown).

Together, the above results suggest that an adult-like topographic map is established before E61 in the feline rubrospinal system.

\section{Synaptic potentials evoked by cortical stimulation in preterm and postnatal rubrospinal neurons}

Having demonstrated that the topography in the rubrospinal projection is established before E61, the location of rubrospinal neurons within the $\mathrm{RN}$ of developing cats could be inferred from their antidromic responses to stimulation of the spinal cord. Thus, by examining the responses to stimulation of different cortical sites in antidromically identified rubrospinal neurons, we were able to study the functional topography in the corticorubral system.

The results described in this and the following sections were obtained from 128 rubrospinal neurons, identified by their antidromic responses to stimulation of $\mathrm{C} 1$ and L1 spinal segments. These neurons had resting membrane potentials more negative than $-50 \mathrm{mV}$, and the fluctuation of resting membrane potential was less than $\pm 2.5 \mathrm{mV}$ during recording. Antidromic action potentials were identified according to previously described criteria (Song et al., 1995b) and are exemplified in Figure $3 A$.

The cruciate sulcus is recognizable from E53 in the cat (Song et al., 1995a). Stimulation of the pericruciate cortex, corresponding to the sensorimotor area in adults, evoked depolarizing po- 

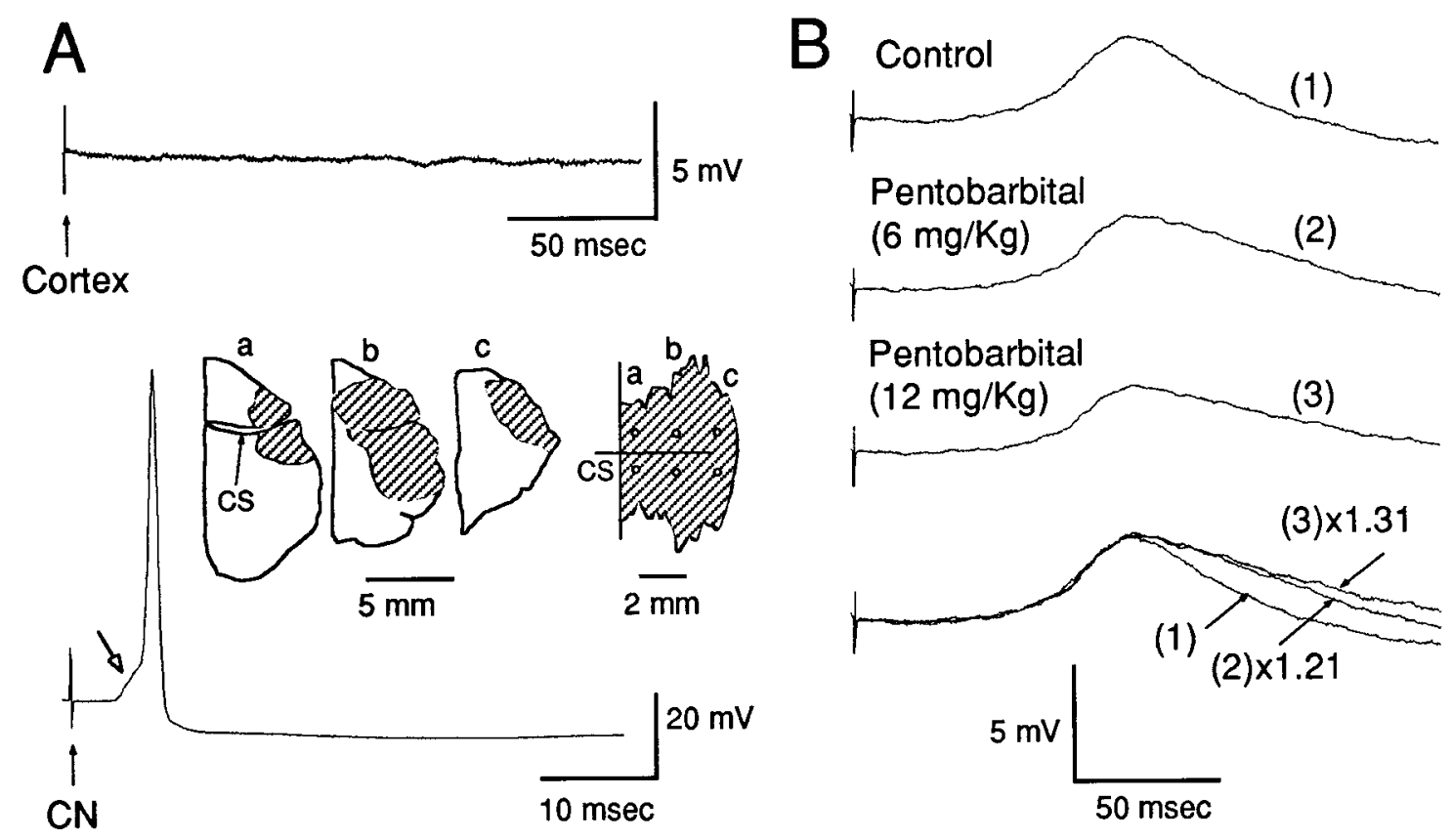

Figure 4. Synaptic potentials evoked by stimulation of the cortex are of cortical origin and are homogeneous up to the peak. $A$, Destruction of cortical neurons eliminated the potentials evoked by cortical stimulation. The figure to the right in the inset depicts the surface view of the pericruciate cortex in which ibotenic acid had been injected in a P4 kitten (injection sites marked by the circles). The rest of the inset figures are drawings of parasagittal sections of the cortex, whose positions are like-labeled in the right figure. Shaded area indicates the area of cortical destruction. Stimulation of such cortical areas at $120 \mathrm{~V}$ evoked no response in rubrospinal neurons (top trace), although in the same cell, both EPSPs and action potentials could be evoked by stimulation of the CN (bottom trace, white arrow points to the EPSP; stimulus strength $=40 \mathrm{~V}$ ). Recordings from a P8 kitten. CS, Cruciate sulcus. $B$, Synaptic potentials evoked by stimulation of the cortex were depressed by intravenous successive application of pentobarbital (top three traces). At the bottom, all three traces were superimposed, with their amplitudes normalized to that of the control, showing the absence of change in shape for potentials from the onset to the peak. All traces are the average of five consecutive recordings from a cell in a P8 kitten. Stimulus strength was $60 \mathrm{~V}$.

tentials in rubrospinal neurons of prenatal (from E61), as well as postnatal, kittens (Fig. 3A). The depolarization was sometimes followed by a hyperpolarization (Fig. $3 A$, bottom trace), which was increasingly common in older animals. The amplitude of the potentials could be gradually changed with varying stimulus strength, suggesting that the potentials are synaptic potentials. Thus, stimulation of the sensorimotor cortex in perinatal kittens evoked EPSPs, sometimes followed by inhibitory postsynaptic potentials, a pattern similar to that seen in adult cats (Tsukahara and Kosaka, 1968). In this study, we focused on the EPSPs, because the EPSP reflects the direct connection between cortical neurons and rubrospinal neurons in adults.

Increasing the strength of stimulation at topographically appropriate cortical sites elicited action potentials at the EPSP peaks in postnatal kittens but never in the preterm kittens. The amplitude of EPSPs evoked with a fixed stimulus strength increased with age (Fig. 3B). Provided that the EPSPs were monosynaptic and of cortical origin, these results suggest that the cortical input from topographically appropriate sites to the RN increases over the perinatal period. Consistent with this notion, the stimulus strength required to evoke an EPSP of fixed amplitude appeared to decrease with age (Fig. 3C).

\section{The EPSPs evoked by cortical stimulation appear to be monosynaptic}

It is most likely that the EPSPs were elicited by activation of efferent neurons in the sensorimotor cortex rather than passing axons. To verify this point, we selectively destroyed cortical neurons by injecting ibotenic acid into the sensorimotor area.
Ibotenic acid selectively destroys cell bodies but leaves axons of passage undamaged, presumably by activating glutamate receptors (Schwarcz et al., 1979). Histological examination of Nisslstained sections revealed that $4 \mathrm{~d}$ after the injection (injected at P4 and examined at P8), virtually no cell bodies were observed within $\sim 2 \mathrm{~mm}$ from the center of injection (Fig. $4 A$, insets). Stimulation of the cortex at intensities up to $120 \mathrm{~V}$ evoked no response in rubrospinal neurons (Fig. $4 A$, top trace), although in the same neuron both EPSP and action potentials could be evoked by stimulation of the contralateral cerebellar nuclei (Fig. $4 A$, bottom trace). Similar results were obtained in all 12 cells in two kittens. These results indicate that the EPSPs evoked in normal kittens derive from neurons of the sensorimotor cortex.

The above experiment, however, does not exclude the possibility that the EPSPs recorded in rubrospinal neurons were elicited polysynaptically. To address this possibility, we first measured the EPSP latencies, defined as the time interval from the stimulus artifact to the time when the potential deflects from the baseline. Although the latency of the EPSPs was longer than that in adults, it did not fluctuate measurably over repeated trials of stimulation. The latencies of all EPSPs are plotted as a function of age in Figure $3 D$. These latencies are comparable to the conduction times of kitten cortical axons from the cortex to the trapezoid body, at corresponding ages (Oka et al., 1985). Because the trapezoid body is further away from the cortex than is the RN, these results support the view that the EPSPs are monosynaptic.

The EPSPs recorded are the temporal and spatial summation of unitary EPSPs of varying latencies (Fig. $3 A$ ). The latency of 

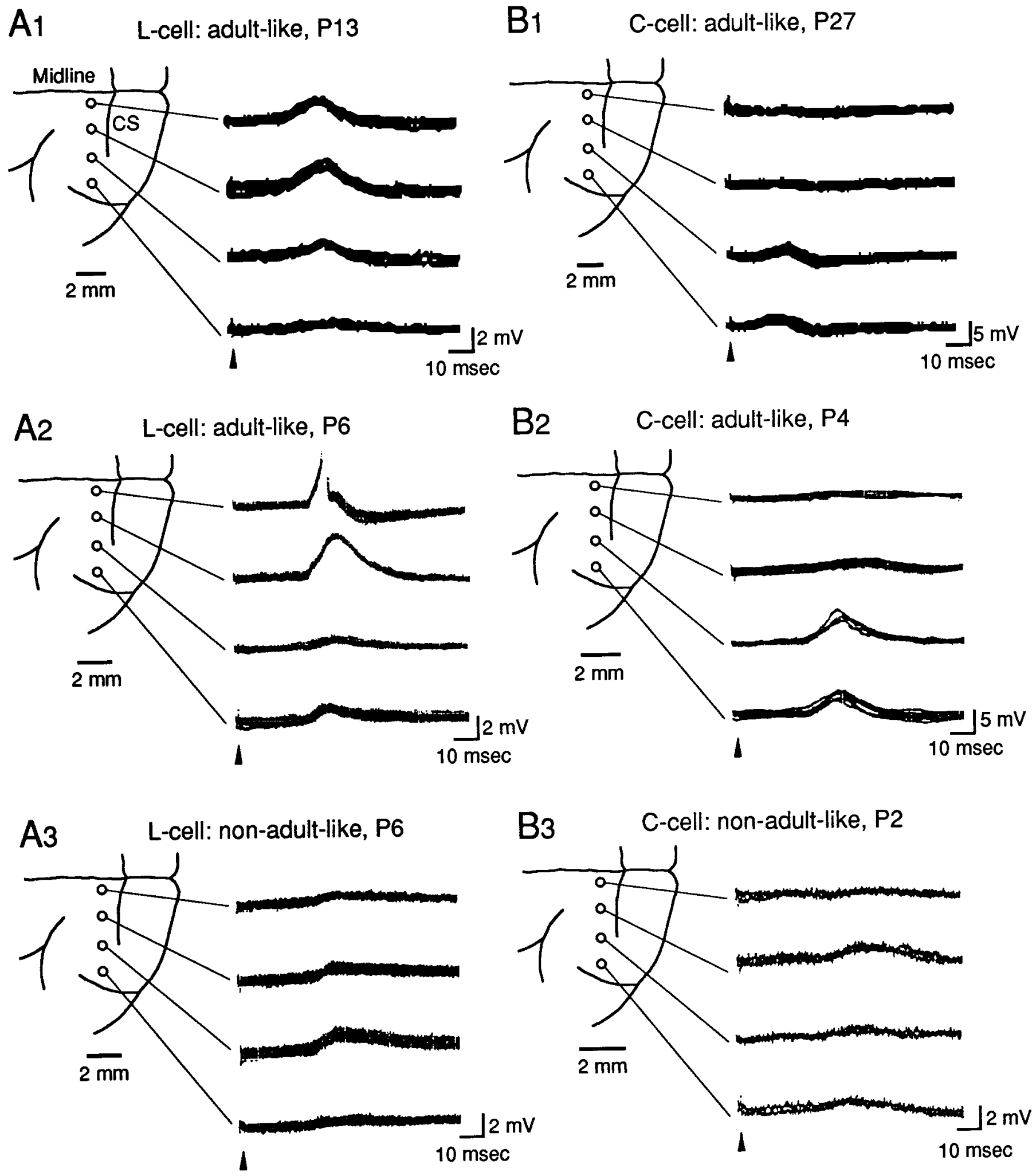

Figure 5. Functional topography of the corticorubral projection in postnatal kittens. A1, The cortical input pattern in an L-cell of a P13 kitten. The cell responded to stimulation of the medial part of the postcruciate gyrus with EPSPs of the largest amplitude, demonstrating that the topographic arrangement in this cell is adult-like. A2, An adult-like L-cell of a P6 kitten. Action potentials were evoked in the top trace but were crippled. A3, An example of an L-cell showing nonadult-like topography in a P6 kitten. B1, A C-cell showing adult-like topography in a P27 kitten. B2, A C-cell showing adult-like topography in a P4 kitten. B3, A C-cell showing nonadult-like topography in a P2 kitten. Stimulus strength was $40 \mathrm{~V}$ for B2 and $60 \mathrm{~V}$ for the rest. All recordings are superpositions of two to five traces. CS, Cruciate sulcus. Scale bars in the insets apply only to the cruciate sulcus. The white circles approximate the positions of stimulation site in the cortex. Arrowheads mark the timing of stimulation. 
such compound EPSPs should reflect the conduction of the fastest axons activated by cortical stimulation. Therefore, the fact that the latency of compound EPSPs coincides with the cortical axonal conduction time does not necessarily indicate that the entire EPSP is monosynaptic. To address this issue, we studied the effect of pentobarbital. In addition to potentiating GABAA receptor function, the general anesthetic pentobarbital is also known to suppress excitatory synaptic transmission (Hubbard et al., 1969). As shown in Figure $4 B$, intravenous injection of pentobarbital suppressed the peak of the EPSP and slowed down its rate of decay (compare the second trace to the first trace). Furthermore, addition of the drug enhanced the effect (Fig. 4B, third trace). In the bottom panel of Figure $4 B$, all traces in panels $1-3$ are superimposed, with the peak amplitude normalized to that of the control recording. It is clear from this figure that the potentials overlap with each other up to the peak, whereas the shape of the potentials after the peak is altered. Similar results were obtained in all five tested neurons in P5-P9 kittens. These results suggest that the component of the EPSPs are homogeneous from the EPSP onset to the peak. Furthermore, because polysynaptic responses are more sensitive to pentobarbital (Hubbard et al., 1969), these results support the notion that the EPSPs are composed of monosynaptic potentials to the peak, although their falling phase may involve polysynaptic ones.

Together, the results described in this section suggest that, under deep anesthesia, stimulation of the sensorimotor cortex evokes monosynaptic EPSPs in rubrospinal neurons of kittens. The amplitude of the EPSPs recorded in rubrospinal neurons thus should reflect the amount of input from the stimulated cortical site.

\section{The pattern of corticorubral connections in postnatal kittens resembles the adult pattern}

The functional topography in the corticorubral projection was thus examined by comparing the amplitude of the EPSPs evoked from the forelimb and the hindlimb areas of the cortex in individual rubrospinal neurons (C-cells and L-cells; see Results, Topography in rubrospinal projections of preterm kittens). In adult cats, L-cells receive input predominantly from the hindlimb cortical region, whereas $\mathrm{C}$-cells receive input primarily from the forelimb region (Tsukahara and Kosaka, 1968). In this study, an L-cell in which the EPSP of the largest amplitude was evoked from the hindlimb region was classified as an L-cell showing adult-like functional topography; otherwise the cell was called nonadult-like. Similarly, a C-cell in which the EPSP of the largest amplitude was evoked from the forelimb region was called adultlike; otherwise, the cell was referred to as nonadult-like. Shown in Figure 5 are representative recordings obtained from postnatal kittens. Figure 5, $A 1$ and $A 2$, shows two examples of L-cells from kittens of different ages. The functional topography in these cells was classified as adult-like, because in these cells the EPSP of the largest amplitude was evoked from the hindlimb area of the cortex. Figure 5, $B 1$ and $B 2$, shows adult-like $\mathrm{C}$-cells receiving the strongest input from the forelimb area, the lateral part of the sensorimotor cortex. In a few cells, however, the topography was nonadult-like. These were L-cells which received the strongest input from the forelimb region (Fig. 5A3) and $\mathrm{C}$-cells receiving the strongest input from the hindlimb area (Fig. 5B3).

In P13-P28 kittens, adult-like topography was observed in 33 of 35 recorded L-cells (94\%) and in six of seven recorded C-cells $(86 \%)$. Although there appeared to be a sampling bias toward L-cells, there was no significant difference in the proportion of cells showing adult-like topography between C-cells and L-cells

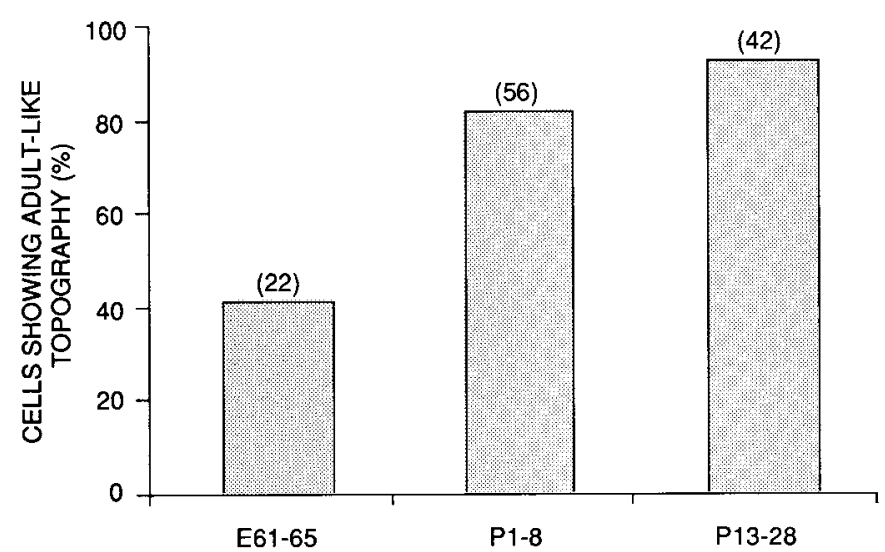

Figure 6. Percentage of neurons exhibiting adult-like functional topography in three groups of different ages. With increasing age, a larger percentage of neurons showed adult-like functional topography. Numbers in parentheses represent the sample size $(n)$ for each group. The percentages of the P1-P8 and P13-P28 groups are significantly $>50 \%(p<$ $0.002)$, whereas the percentage of the E61-E65 group is not $(p>0.4)$.

$(p>0.4)$. We thus pooled the results of $\mathrm{C}$-cells and L-cells together. In P13-P28 kittens, 93\% (39 of 42) of recorded rubrospinal neurons showed adult-like functional topography (Fig. 6).

In P1-P8 kittens, cells showing adult-like topography constituted $82 \%$ of the total ( $n=56$; C-cell, 9 of 11 ; L-cell, 37 of 45 ) (Fig. 6). This percentage was not significantly different from that of the P13-P28 kittens $(p>0.1)$. For both groups, the percentages cannot be expected from a random corticorubral connection $(p<0.002)$, in which case the probability of observing adult-like cells would be $50 \%$. These results suggest that the functional topography in the corticorubral system is present in the first postnatal week.

\section{Corticorubral connection in preterm kittens is diffuse}

The small-sized brain of preterm kittens allowed us to set stimulating electrodes at only two sites in the cortex (see Materials and Methods). In all preterm kittens, stimulation of either site evoked responses of similar amplitudes in rubrospinal cells, as exemplified in Figure 7. The topography of each cell was nevertheless classified either as adult-like or nonadult-like, following the definition described above. The L-cell shown in Figure $7 A$, for example, was classified as a cell showing adult-like topography, because the amplitude of the EPSP evoked from the hindlimb region was slightly larger; the L-cell shown in Figure $7 B$ was classified as nonadult-like, because the amplitude of the EPSP evoked from the hindlimb region was smaller. In contrast to postnatal kittens, of all 22 rubrospinal neurons recorded in E61E65 preterm kittens, only $41 \%$ showed adult-like functional topography (C-cell, 2/4; L-cell, 7/18) (Fig. 6). This percentage is not significantly different from the probability of $0.5(p>0.4)$, which would be expected if the corticorubral innervation were random. These results suggest that the functional corticorubral connection in preterm kittens is diff use.

The result that the amplitudes of the EPSPs evoked from the two stimulation sites were comparable raises the possibility that the stimulation current had spread to the neighboring stimulation site so that both electrodes stimulated an overlapping population of efferent neurons. To test this, both sites were stimulated simultaneously with the same strength as the case when each site was stimulated alone (Fig. 7A). The amplitude of EPSPs evoked in this manner was close to the summation of the EPSPs evoked 
from each of the two sites (Fig. 7A, bottom panel), suggesting that the response evoked from each site primarily reflects the cortical input from that site. Similar results were obtained in all tested cells in prenatal $(n=5)$ and postnatal kittens $(n=8)$ with subthreshold stimulation.

Because the corticorubral projection in preterm kittens was assessed with two pairs of electrodes and that in postnatal kittens was done with four pairs, the difference in the observed pattern of connection between the two groups could have been attributable to the methodological difference. To address this possibility, three P5-P7 kittens were studied with two pairs of stimulating electrodes, set in the same manner as in the preterm kittens. Seven of eight neurons $(87.5 \%)$ recorded from these kittens showed adultlike functional topography, a result comparable to that obtained with four pairs of electrodes in P1-P8 kittens.

\section{DISCUSSION}

We have examined the development of the pattern of functional inputs from the cortex to identified rubrospinal neurons in the cat. To our knowledge, this is the first study concerning the development of functional topography in mammals using synaptic potentials recorded intracellularly in vivo. Our results indicate that the establishment of topography in the corticorubral system starts from a phase of diffuse functional connections before birth, suggesting that cortical axons initially form synapses nonselectively with rubrospinal neurons. The functional topography emerges by the first postnatal week, well before the establishment of an anatomical map, which appears only at approximately P13 by selective axon arborization in topographically appropriate regions (Higashi et al., 1990). These results suggest that selective promotion of synapse formation and/or selective enhancement of synaptic efficacy occurs before development of cortical axon branches in topographically appropriate regions in the RN.

\section{EPSP amplitude as an index for studying the development of functional topography}

Stimulation of the cortex evoked potentials in rubrospinal neurons in both the fetuses and the neonates. These potentials may or may not be mediated by synapses having the mature type of membrane specializations (Buchanan et al., 1989). We nevertheless call these synaptic potentials.

At early stages of development, synaptic activity is expected to be subthreshold, as demonstrated in the present study. The corticorubral synaptic activity in the fetal cats would therefore not have been detected with extracellular-recording based techniques. Thus, although technically challenging, it is necessary to record synaptic potentials rather than unit activity to study early development of functional neuronal connectivity.

To use cortically evoked EPSPs to assess the topography of corticorubral connections, it is essential to isolate monosynaptic EPSPs. In principle, the most compelling evidence for a monosynaptic EPSP is that its latency, i.e., the sum of conduction time of the presynaptic axon and a synaptic delay, is less than two synaptic delays. Immature axons, however, have low conduction velocities and thus long conduction times (Song et al., 1995b; Olivier et al., 1997), making it difficult to judge whether observed synaptic potentials are monosynaptic. The issue is further complicated by the fact that there are considerable variations in conduction velocity, even among axons in a single group of neurons during development (Oka et al., 1985; Song et al., 1995b). Nevertheless, the results presented here together support the view that the rising phase of the cortically evoked potentials was

\section{A L-cell: adult-like, E62}

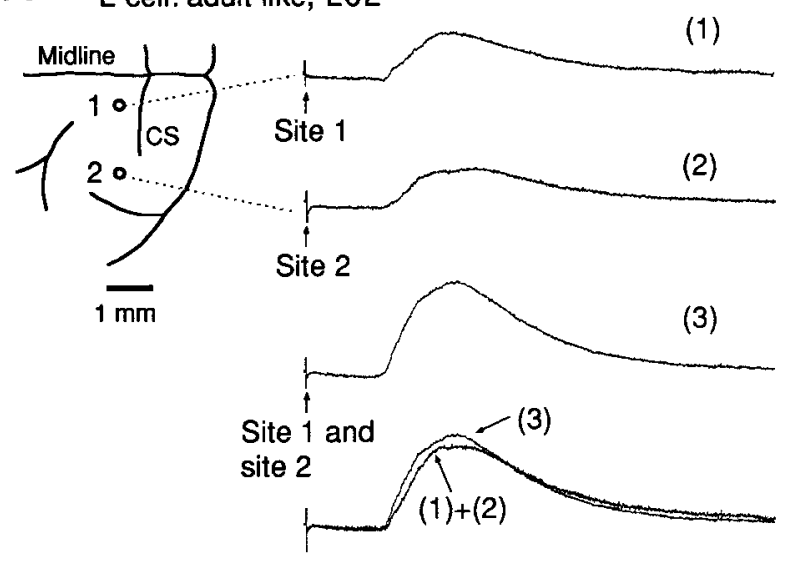

B

L-cell: non-adult-like, E62

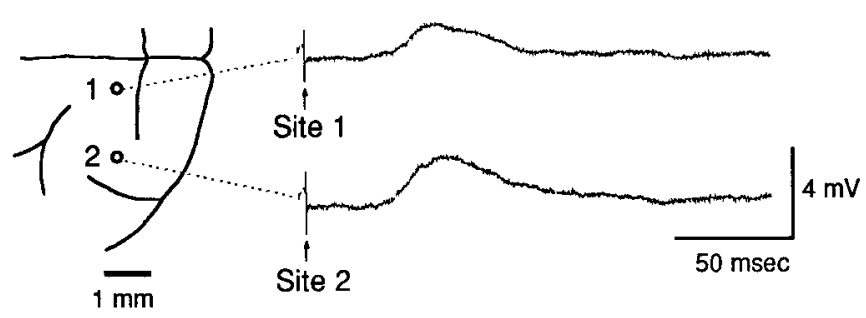

Figure 7. Functional topography of the corticorubral projection in fetal cats. $A$, The responses of an L-cell in an E62 fetus to stimulation of the two cortical sites are shown as the top two traces. This cell was classified as adult-like, because the response evoked from the hindlimb region was larger. Stimulus strength was $80 \mathrm{~V}$. The trace labeled as (3) is the response evoked by simultaneous stimulation of both cortical sites. At the bottom, this recording was superimposed to the sum of the EPSPs evoked by stimulation of each of the two sites. The slightly larger amplitude of the (3) recording than that of the sum suggests that the stimulation to the two sites overlapped only at subthreshold level. $B$, An example of an L-cell showing nonadult-like topography in an E62 fetus. Stimulus strength was $50 \mathrm{~V}$. All traces are averages of three consecutive recordings. CS, Cruciate sulcus. Scale bars in the insets apply only to the cruciate sulcus. Calibration in $B$ also applies to $A$.

composed of monosynaptic EPSPs. Our previous electron microscopic finding that cortical axons make synapses with RN neurons in newborn kittens also supports this view (Saito et al., 1997).

EPSP amplitude should reflect the number of functional synapses activated by the cortical stimulation. However, because most of the EPSPs were of complex shape, the total charge transfer might be a better index for estimating the number of input synapses. We thus compared the results using either EPSP amplitude or the integration from the onset to the peak of the EPSPs in 10 randomly selected neurons, but no difference was found. We therefore used EPSP amplitude for all cells for the ease of measurement.

\section{Proliferative versus regressive mechanisms for the formation of topography in the corticorubral connection}

Cells showing adult-like functional topography accounted for only approximately half of the cells in the preterm kittens, whereas their proportion increased to $>90 \%$ over the first postnatal month. These results indicate that functional topography in the corticorubral pathway does not exist at the early stage of 


\section{Fetus}

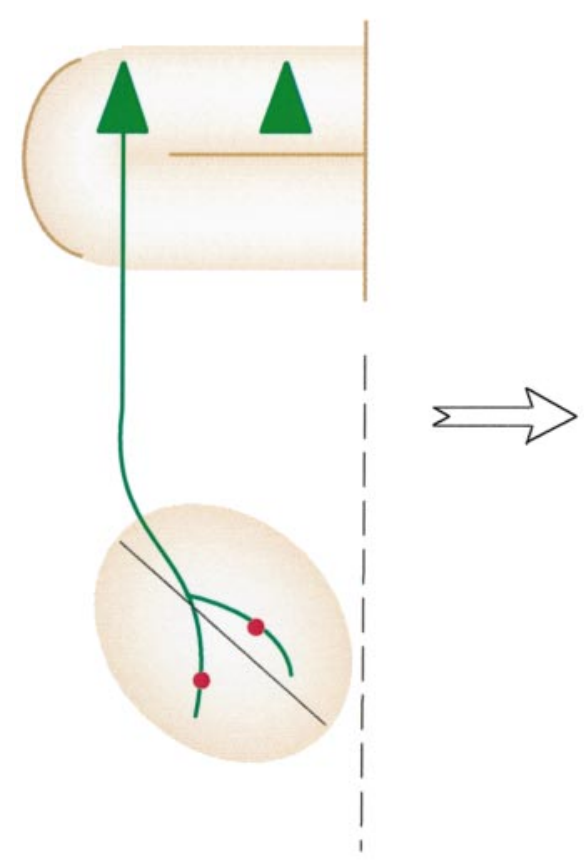

Neonate

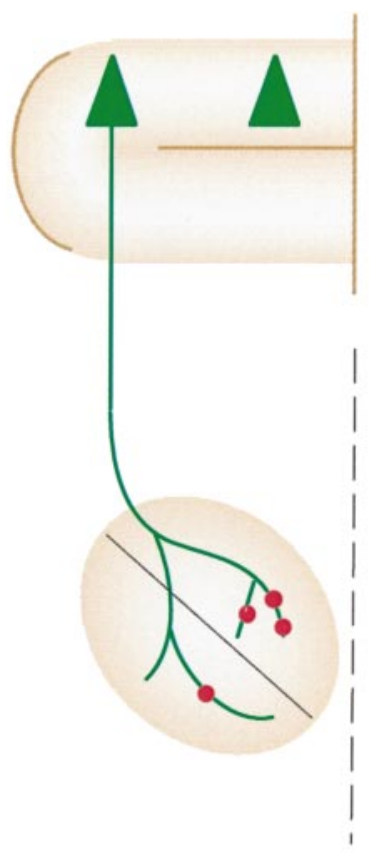

Adult

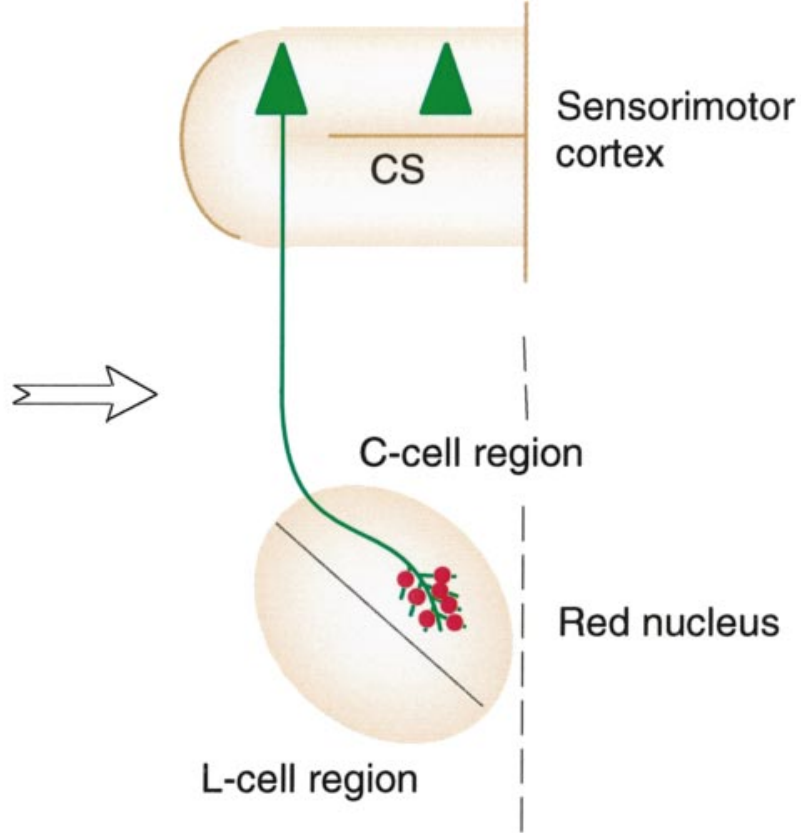

Midline

Figure 8. A schematic summary of the development of functional and anatomical topographic maps in the corticorubral system. The anatomical part is from Higashi et al. (1990). For clarity, only the cortical projection from the forelimb area is shown. Red spots represent functional synapses or synapses of high synaptic efficacy. In fetuses, neither the anatomical map nor the functional map exists. In neonates, however, a functional map appears despite the absence of an anatomical map. Comparison of the development of these two kinds of maps suggests that cortical axons form functional synapses nonselectively with rubrospinal neurons before birth. Furthermore, the earlier emergence of the functional map suggesting that selective promotion of synapse formation and/or selective enhancement of synaptic efficacy in topographically appropriate regions occurs before the mature anatomical map is formed. $C S$, Cruciate sulcus.

cortical innervation but appears to be formed over the period of early postnatal life. The absence of functional topography in the preterm kittens suggests that cortical axons are not strictly guided to topographically correct regions after ingrowth into the RN. This view is consistent with our previous anatomical observations that focally labeled cortical neurons have their axons throughout the RN in preterm cats (Song et al., 1995a). One possible cellular mechanism for the developmental increase in the percentage of cells showing adult-like functional topography is selective elimination of synapses in topographically inappropriate regions. The idea that selective elimination, or conversely, selective stabilization of synapses or axon terminals works as a mechanism for pattern formation has been proposed for many years (Changeux and Danchin, 1976; Hubel et al., 1977). In the corticorubral projection, however, the cortical inputs from the topographically inappropriate cortical regions do not seem to be eliminated during the period of topography formation, because stimulation of these regions evoked EPSPs in postnatal kittens in which the corticorubral topographic map is already present (Fig. 5A2). Moreover, the increase in EPSP amplitude accompanying the formation of the functional topography (Fig. 3) is hard to explain by regressive mechanisms. A more likely possibility is selective proliferation of synapses or selective enhancement of synaptic efficacy in topographically appropriate regions. The proliferation of synapses can be achieved by an increase in the number of synapses on existing fibers in a region (also see below) or by local elaboration of axonal branches. Developmental increase in branch number of cortical axons has been reported previously (Higashi et al., 1990; Song et al., 1995a), although this increase does not appear to be region-specific until 2 weeks after birth (Higashi et al., 1990). There is now an increasing body of evidence supporting the predominant role of selective construction of axonal branches over selective elimination in the formation of adult-like connection patterns (Armand et al., 1997; for review, see Murakami et al., 1992; Purves et al., 1996).

An alternative cellular mechanism for the formation of topography in the corticorubral projection would be that all cortical axons do not enter the $\mathrm{RN}$ at the same time, and axons arriving after birth project preferentially to topographically appropriate $\mathrm{RN}$ regions. This possibility, however, seems unlikely, because in newborn kittens cortical axons with growing tips were observed in the RN but not along the pathways of the corticorubral projection (our unpublished observation).

\section{Selective formation of synapse as a mechanism for formation of patterned neuronal connectivity}

We have shown previously that cortical axons in the RN do not show localized distribution in prenatal cats (Song et al., 1995a). The currently demonstrated absence of functional topography in the preterm cats would thus suggest that cortical axons make functional connections nonselectively with rubrospinal neurons before birth. In other words, the axons are able to make functional synapses with neurons in both topographically appropriate and inappropriate regions. By inference from the findings in 
rodent retinotectal projections that early developing retinal axons showing no preferential termination in vivo do respond to tectumderived positional cues in vitro (Simon and O'Leary, 1992a,b), one may suppose the presence of positional cues in the RN of fetal cats. Such cues, however, apparently do not instruct cortical fibers to form synapses in a region-specific manner in vivo after growth into the RN, although they may do so at a later stage of development. Developing axons in many other regions of the brain have also been shown to be highly tolerant in selecting target cells for forming synapses (Takeda and Maekawa, 1989; for review, see Purves and Lichtman, 1985). An exception, however, has been documented in the spinal cord in which spindle afferents make functional synapses preferentially with homonymous motoneurons from the outset of development (Frank and Westerfield, 1983; Mendelson and Frank, 1991; Rafuse et al., 1996; Mears and Frank, 1997).

It is surprising that $>80 \%$ of cells in P1-P8 kittens showed adult-like functional topography, because, anatomically, the corticorubral projection does not exhibit a clear topography until P13 (Higashi et al., 1990) (Fig. 8). As discussed above, the increase in the percentage of cells showing adult-like functional topography over the perinatal period may be attributable to enhancement of functional input from topographically appropriate cortical regions, as evidenced by the increase in EPSP amplitude (Fig. 3B). Thus, the apparent discrepancy between the previous anatomical and the present electrophysiological results concerning the early development of topography can be explained by two mutually nonexclusive possibilities. One is that cortical axons form more functional synapses in topographically appropriate $\mathrm{RN}$ regions than in inappropriate regions during the first 2 postnatal weeks; the other is that, during this period, synapses formed by cortical axons in topographically appropriate RN regions have enhanced synaptic efficacy compared with those in the inappropriate regions (Fig. 8). In any case, our results thus suggest that there is a developmental step of selective promotion of the formation of functional synapses and/or selective enhancement of synaptic efficacy in topographically appropriate regions before proliferation of axon branches proceeds in the region. Identifying molecular mechanisms facilitating formation of functional synapses or enhancing synaptic efficacy in topographically appropriate regions in future experiments would be of crucial value for understanding the formation of topographic maps in mammals.

Although cortical axons arrive at the RN by E50 (Song et al., 1995a), functional topography began to form only during the first postnatal week. This raises the possibility that the signal that instructs the formation of the topographic map may not be available until approximately the time of birth. Such delayed expression of the signal may rely on intrinsic genetic programs. Alternatively, the expression might be triggered by neuronal activity, which is expected to be increased by sensory input after birth (Stryker, 1989; Shatz, 1990).

\section{Functional significance}

Under the influence of the sensorimotor cortex and the cerebellar deep nuclei, rubrospinal neurons are primarily involved in motor control (Kohlerman et al., 1982). Because the somatotopy of sensory afferents to the cortex is already established at birth (Rubel, 1971), the functional topography in the corticorubral projection observed from the first postnatal week would provide a basis for sensory information in the cortex to be integrated into the rubrospinal pathway in a topographic manner. Although corticorubral fibers continue to develop after the first postnatal week (Higashi et al., 1990), the functional topography in the corticorubrospinal system in newborn kittens would enable the system to contribute to the coordination of behavior during early postnatal life, before the corticorubral system is fully developed.

\section{REFERENCES}

Armand J, Olivier E, Edgley SA, Lemon RN (1997) Postnatal development of corticospinal projections from motor cortex to the cervical enlargement in the macaque monkey. J Neurosci 17:251-266.

Buchanan J, Sun Y-A, Poo M-M (1989) Studies of nerve-muscle interaction in Xenopus cell culture: fine structure of early functional contacts. J Neurosci 9:1540-1554.

Changeux J-P, Danchin A (1976) Selective stabilization of developing synapses as a mechanism for the specification of neuronal networks. Nature 264:705-712.

Drescher U, Bonhoeffer F, Muller BK (1997) The Eph family in retinal axon guidance. Curr Opin Neurobiol 7:75-80.

Frank E, Westerfield M (1983) Development of sensory-motor synapses in the spinal cord of the frog. J Physiol (London) 343:593-610.

Gierer A, Muller CM (1995) Development of layers, maps and modules. Curr Opin Neurobiol 5:91-97.

Godement P, Bonhoeffer F (1989) Cross-species recognition of tectal cues by retinal fibers in vitro. Development 106:313-320.

Higashi S, Yamazaki M, Murakami F (1990) Postnatal development of crossed and uncrossed corticorubral projection in kitten: a PHA-L study. J Comp Neurol 299:312-326.

Hubbard JI, Llinas R, Quastel DMJ (1969) Electrophysiological analysis of synaptic transmission. London: Arnold.

Hubel DH, Wiesel TN, LeVay S (1977) Plasticity of ocular dominance columns in monkey striate cortex. Philos Trans R Soc Lond B Biol Sci 278:377-409.

Jeneskog T, Padel Y (1983) Cerebral cortical areas of origin of excitation and inhibition of rubrospinal cells in the cat. Exp Brain Res 50:309-320.

Kohlerman NJ, Gibson AR, Houk JC (1982) Velocity signals related to hand movements recorded from red nucleus neurons in monkeys. Science 217:857-860.

Lapin LL (1983) Probability and statistics for modern engineering. Boston: $\mathrm{B} / \mathrm{C}$ Engineering Division.

Mabuchi M, Kusama T (1966) The corticorubral projection in the cat. Brain Res 2:254-273.

Mears SC, Frank E (1997) Formation of specific monosynaptic connections between muscle spindle afferents and motoneurons in the mouse. J Neurosci 17:3128-3135.

Mendelson B, Frank E (1991) Specific monosynaptic sensory-motor connections in the chick embryonic spinal cord form in the absence of patterned neuronal activity and motoneuronal cell death. J Neurosci 11:1390-1403.

Murakami F, Song W-J, Katsumaru H (1992) Plasticity of neuronal connections in developing brains of mammals. Neurosci Res $15: 235-253$

Oka H, Samejima A, Yamamoto T (1985) Postnatal development of pyramidal tract neurons in kittens. J Physiol (Lond) 363:481-499.

Olivier E, Edgly SA, Armand J, Lemon RN (1997) An electrophysiological study of the postnatal development of the corticospinal system in the macaque monkey. J Neurosci 17:267-276.

Pompeiano O, Brodal A (1957) Experimental demonstration of a somatotopical origin of rubrospinal fibers in the cat. J Comp Neurol 108:225-252.

Purves D, Lichtman JW (1985) Principles of neural development. Sunderland, MA: Sinauer.

Purves D, White LE, Riddle DR (1996) Is neural development Darwinian? Trends Neurosci 19:460-464.

Rafuse VF, Milner LD, Landmesser LT (1996) Selective innervation of fast and slow muscle regions during early chick neuromuscular development. J Neurosci 16:6864-6877.

Roskies A, O’Leary DDM (1994) Control of topographic retinal axon branching by inhibitory membrane-bound molecules. Science 265:799-803.

Roskies A, Friedman GC, O'Leary DDM (1995) Mechanisms and molecules controlling the development of retinal maps. Perspect Dev Neurobiol 3:63-75.

Rubel EW (1971) A comparison of somatotopic organization in sensory 
neocortex of newborn kittens and adult cats. J Comp Neurol 143:447-480.

Saito Y, Song W-J, Murakami F (1997) Preferential termination of corticorubral axons on spine-like dendritic protrusions in developing cat. J Neurosci 17:8792-8880.

Sakaguchi DS, Murphey RK (1985) Map formation in the developing Xenopus retinotectal system: an examination of ganglion cell terminal arborizations. J Neurosci 5:3228-3245.

Schwarcz R, Hokfelt T, Fuxe K, Jonsson G, Goldstein M, Terenius L (1979) Ibotenic acid-induced neuronal degeneration: a morphological and neurochemical study. Exp Brain Res 37:199-216.

Shatz CJ (1990) Impulse activity and the patterning of connections during CNS development. Neuron 5:745-756.

Simon DK, O'Leary DDM (1992a) Development of topographic order in the mammalian retinocollicular projection. J Neurosci 12:1212-1232.

Simon DK, O'Leary DDM (1992b) Responses of retinal axons in vivo and in vitro to position-encoding molecules in the embryonic superior colliculus. Neuron 9:977-989.

Soeda T, Ohta M, Ohmatsu S (1980) Principles and applications of statistics. Tokyo, Japan: Nisshin.

Song W-J, Kobayashi Y, Murakami F (1993) An electrophysiological study of a transient ipsilateral interpositorubral projection in neonatal cats. Exp Brain Res 92:399-406.

Song W-J, Kanda M, Murakami F (1995a) Prenatal development of cerebrorubral and cerebellorubral projections in cats. Neurosci Lett 200:41-44.

Song W-J, Okawa K, Kanda M, Murakami F (1995b) Perinatal development of action potential propagation in cat rubrospinal axons. J Physiol (Lond) 488:419-426.

Stirling RV (1991) Molecules, maps and gradients in the retinotectal projection. Trends Neurosci 14:509-512.

Stryker MP (1989) Role of neural activity in development and plasticity of mammalian visual cortex. Biomed Res [Suppl] 10:37-42.

Stuermer CAO (1988) Retinotopic organization of the developing retinotectal projection in the zebrafish embryo. J Neurosci 8:4513-4530.

Takeda T, Maekawa K (1989) Transient direct connection of vestibular mossy fibers to the vestibulocerebelar purkinje cells in early postnatal development of kittens. Neuroscience 32:99-111.

Tsukahara N, Kosaka K (1968) The mode of cerebral excitation of red nucleus neurons. Exp Brain Res 5:102-117.

Udin SB, Fawcett JW (1988) Formation of topographic maps. Annu Rev Neurosci 11:289-327.

Vielmetter J, Stuermer CA (1989) Goldfish retinal axons respond to position-specific properties of tectal cell membranes in vitro. Neuron 2:1331-1339.

Walter J, Kern-Veits B, Huf J, Stolze B, Bonhoeffer F (1987) Recognition of position-specific properties of tectal cell membranes by retinal axons in vitro. Development 101:685-696. 